\title{
Réflexion sur les fondements juridiques du principe d'optimisation de la radioprotection*
}

\author{
M.C. GRENERY-BOEHLER, J. LOCHARD**
}

(Manuscrit reçu le 20 septembre 1991)

RÉSUMÉ Les disciplines juridiques tendent, en général, à une rationalisation et à une stabilisation des pratiques sociales ou économiques et elles s'orientent vers des concepts ou des pratiques se rattachant au domaine du déterminé. En ce qui concerne le principe d'optimisation de la radioprotection, la technique juridique classique du droit administratif ne répond pas exactement aux problèmes posés par sa mise en cuvre : des obligations de prestations imposées traditionnellement par l'administration, il semble qu'il faille passer à une obligation de comportement des acteurs, d'où la difficile qualification juridique du principe d'optimisation. Du droit du commandement, du privilège et du contrôle, l'administration doit, dans le cas de l'optimisation, faire essentiellement confiance aux exploitants des installations nucléaires par l'édiction d'une "norme-objectif" et non plus d'une "norme-règle". L'avenir des sciences juridiques n'est-il pas dans le développement du droit administratif du non déterminé qui obligerait l'administration à reconnaître que, même dans le domaine du déterminé, elle n'est pas toujours celle qui sait ? Si notre droit administratif classique est un droit du commandement et du contrôle, le droit administratif du non déterminé sera celui du droit de l'effort commun, encadré par des actes collectifs avec pour forfait la confiance, la concertation, les obligations de comportement, le tout sous le contrôle d'un juge intervenant lorsqu'il y a contradiction manifeste entre les actes et le comportement promis.

En droit français, l'optimisation est restée un principe général non assorti de dispositions concrètes quant aux modalités de mise en œuvre. Cet article a pour objectif d'explorer sur quels fondements juridiques il serait possible d'appliquer, sur le plan pratique, ce principe sans en trahir l'esprit.

ABSTRACT As a general rule, the different branches of the legal profession have a tendency to rationalize and stabilize social or economic practices and to be inclined towards concepts or practices belonging to the field of the definite. With respect to the principle of optimization for radiological protection, conventional legal procedures in administrative law do not entirely cover the problems raised by its implementation : from the obligation to provide a service, generally required by the public administration, it would appear we have to change to a guarantee of actors' behaviour, hence the difficulty in legally qualifying the principle of optimization. As for the law of authority, privilege and control, in the case of optimization the public administration must basically trust nuclear plant operators by drawing up a "standard objective" rather than a "standard rule". Does not the future of legal sciences lie in developing administrative law for the field of the

\footnotetext{
* Communication présentée lors du colloque de l'Association internationale de droit nucléaire (AIDN/INLA), "Le droit nucléaire et l'énergie nucléaire de demain", Bath, UK, 23-26 septembre 1991.

** Centre d'étude sur l'évaluation de la protection dans le domaine nucléaire (CEPN), BP 48, 92263 Fontenay-aux-Roses Cedex, France.
} 
indefinite, thereby forcing public administration to recognize that even in the field of the definite, it is not always infallible ? If our conventional administrative law is a law of authority and control, administrative law for the field of the indefinite must be one of common efforts within a context of community actions requiring trust, agreement and guaranteed behaviour, falling under a judge's control whenever there is obvious contradiction between acts and the promised behaviour.

Under the French law, optimization has remained a general principle with no corresponding concrete provisions for its implementation. The purpose of this paper is to explore on which legal bases the optimization principle could be applied practically without betraying its actual spirit.

\section{La gestion de la radioprotection et le principe d'optimisation}

\subsection{Rappel du système de limitation des doses recommandé par la Commission internationale de protection radiologique (CIPR)}

La CIPR a approfondi et précisé sa doctrine concernant la protection de l'homme contre les rayonnements ionisants en adoptant la Publication 26 en 1977 et plus récemment la Publication 60 en avril 1991 [4, 7].

La Publication 26 comportait deux impératifs : d'une part, prévenir toute apparition d'effets non stochastiques (non aléatoires), c'est-à-dire d'effets pour lesquels on sait qu'il existe une dose seuil au-dessous de laquelle ces effets n'apparaissent jamais (érythème, cataracte, etc...), d'autre part, limiter à un niveau acceptable pour l'individu et pour la société le détriment éventuel pour la santé que pourraient entraîner les effets stochastiques (ou aléatoires) c'est-à-dire les effets qui n'apparaissent que chez quelques-uns des individus irradiés et ceci au hasard (leucémies, cancers, effets génétiques...).

Etant incapable de préciser la forme exacte de la relation entre la dose et la probabilité d'apparition de ces derniers effets à faible dose, la CIPR a retenu l'hypothèse d'une relation linéaire et sans seuil considérée comme prudente au niveau des faibles doses. Sur la base de cette exposition-risque, la CIPR a développé un système complet de gestion du risque articulé autour de trois principes fondamentaux :

1) aucune pratique ne peut être autorisée sans que son introduction ne produise un bénéfice positif net : c'est le principe de justification des activités ;

2) toutes les expositions doivent être maintenues au niveau le plus bas que l'on pourra raisonnablement atteindre, compte tenu des facteurs économiques et sociaux : c'est le principe d'optimisation de la protection ;

3) l'équivalent de dose reçu par les individus ne doit pas dépasser les limites recommandées par la CIPR : c'est le principe de limitation des doses individuelles. 
Bien que reposant sur les mêmes bases que précédemment, la Publication 60 marque, cependant, quelques évolutions notables quant à la philosophie de la gestion du risque :

- l'extension du système (justification, optimisation et limitation des doses) aux expositions probabilistes et non plus seulement certaines ;

- la distinction entre la gestion des sources contrôlables et les situations dites de facto;

- l'introduction du principe d'équité au niveau de la gestion des doses individuelles. En effet, l'utilisation de l'approche collective, en particulier au niveau du principe d'optimisation, n'assure pas toujours une protection équitable des individus dans la population exposée, et il devient alors nécessaire d'introduire certains correctifs pour mieux répartir les expositions individuelles. Sur le plan pratique, cette innovation se traduit par l'introduction du concept de "contrainte" de dose individuelle.

\subsection{La place de l'optimisation}

La principe d'optimisation, repris sous l'acronyme ALARA (As low as reasonably achievable), dans la terminologie de la CIPR, est progressivement devenu au fil des publications de cette dernière un principe fondamental de son système de limitation des doses [14]. Ce dernier ne s'appuie plus exclusivement, comme précédemment, sur des doses maximales admissibles utilisées comme limite supérieure du risque acceptable. La limite est maintenant considérée comme la frontière la plus basse de la région des doses inacceptables. Les valeurs au-dessus de la limite doivent être interdites réglementairement, et les doses en dessous de la limite ne sont considérées comme tolérables et acceptables que dans la mesure où les niveaux d'exposition résiduels sont optimisés.

Se trouve ainsi affirmé le principe selon lequel, certains plafonds de doses étant fixés (en règle générale, et selon les recommandations de la CIPR, $20 \mathrm{mSv}$ par an en moyenne sur 5 ans pour les travailleurs exposés et $1 \mathrm{mSv}$ pour les personnes du public), ces plafonds ne sauraient être considérés comme des limites "autorisées" mais, au contraire, comme des maximums dont il faut, dans la mesure du raisonnable, s'éloigner. Les limites ne doivent pas être considérées comme incitatives mais comme dissuasives.

\subsection{La formalisation}

L'optimisation de la radioprotection comprise comme la recherche d'un arbitrage entre les coûts de protection et les niveaux d'exposition résiduels apparaît donc comme une tentative de fonder le problème du choix de l'allocation des ressources de protection sur un critère socialement accepté. II s'agit là, en fait, d'une réponse à ceux qui prônent l'argument du "risque nup' et ce faisant, oublient qu'au-delà d'un certain niveau de risque résiduel, toute amélioration peut aller à l'encontre d'une allocation rationnelle des ressources de protection [9]. 
Si l'on veut éviter que le mouvement irréversible vers une protection toujours accrue des populations n'aboutisse à une dilapidation des ressources disponibles, il faut alors reconnaître au principe d'optimisation l'intérêt que ce type de démarche présente comme outil d'aide à la décision au niveau des choix fondamentaux de radioprotection, tant à la conception que pendant l'exploitation des installations [18].

Il convient de garder à l'esprit que l'application du principe d'optimisation ne saurait être réduite à la seule mise en œuvre de méthodes particulières d'aide à la décision telle que, par exemple, l'analyse coût-bénéfice qui a été très tôt suggérée par la CIPR $[5,6]$. L'optimisation peut tout aussi bien faire l'objet d'une approche pragmatique faite d'intuition et de bon sens. Pour les praticiens, les gens du "terrain", le principe d'optimisation est une invite constante à étudier les projets, planifier et préparer les interventions avec le souci de réduire les doses susceptibles d'être reçues pour le personnel ou les tiers en tenant compte des limites techniques et économiques. De ce point de vue, la démarche du principe d'optimisation est un état d'esprit qui doit animer les responsables et les acteurs de la radioprotection au niveau de la conception et de l'exploitation des installations. Si l'emploi de techniques décisionnelles formalisées peut apporter des éclairages très utiles pour élaborer des politiques de protection rationnelles, il apparaît, sur le plan juridique, que la transposition de ces techniques en réglementation est impossible et que l'optimisation doit rester un simple rappel de principe s'opposant à une formalisation incapable de rendre compte de la spécificité des situations particulières.

\section{La nécessaire mais insuffisante radioprotection de contrôle}

Alors que l'effort de la CIPR porte sur le principe d'optimisation, les limites de dose jouant essentiellement un rôle correcteur et subsidiaire, c'est paradoxalement cette fixation des limites qui est la plus développée dans la réglementation. Dans la plupart des législations, la transposition juridique des principes de justification et d'optimisation est une reprise littérale de la formulation initiale de la CIPR. Le contrôle de l'application de ces principes est laissé à l'appréciation discrétionnaire des autorités et peut s'exercer dans le cadre des procédures classiques de police administrative : autorisations de création et d'exploitation, inspections...

En fait, une réglementation contraignante de la radioprotection fondée sur la notion de seuil juridique établie sur les doses maximales admissibles semble garder la faveur des pouvoirs publics qui fonctionnent essentiellement dans une approche de type "juridique et réglementaire" [2]. Cette démarche traditionnelle, conforme au droit administratif classique, implique l'édiction de normes-règles qui prévoient des dispositions de nature préventive de façon à éviter la violation de ces normes.

Dans le cadre de la radioprotection, il est d'usage de distinguer deux domaines en matière de réglementation et de pratiques [10]. Le premier 
concerne ce qu'il convient d'appeler, en général, les normes fondamentales ou normes de base, c'est-à-dire l'élaboration des limites supérieures des risques ou des nuisances considérées comme acceptables dans l'état "actuel" des connaissances sur les effets. Le second domaine en matière de réglementation et de pratiques peut être appelé conventionnellement celui des normes dérivées et des pratiques qui en découlent : les procédures de déclaration préalable, d'autorisation préalable voire d'interdiction des sources ou activités impliquant les expositions (contrôle "indirect" ou contrôle au sens large) ainsi que les inspections (contrôle "direct" ou au sens strict) de ces sources ou activités autorisées et la surveillance médicale.

Ainsi, les systèmes de déclaration et d'autorisation et le droit d'inspection sont les éléments les plus importants de la réglementation traditionnelle en matière nucléaire : ce sont eux qui permettent d'assurer en permanence le respect des normes prescrites. II s'agit donc de privilégier des règles sûres et de limiter la part d'incertitude qu'il y a nécessairement dans le déroulement des actions humaines. En effet, les disciplines juridiques tendent, en général, à une rationalisation et à une stabilisation des.phénomènes sociaux ou économiques et elles s'orientent vers des concepts ou des techniques se rattachant au droit du commandement, du privilège et du contrôle. La réglementation administrative est contraignante et se résout, en fait, en une émission d'ordres à l'intention des administrés qui suppose une obligation de respect absolu des normes [13, 23].

Par l'édiction d'une norme-règle, l'Etat cherche à assurer la sécurité individuelle et collective des citoyens par un degré suffisant de stabilité et de force contraignante du droit et de ses instruments de mise en œuvre. De plus, la norme-règle présente l'avantage d'une méthode efficace assortie de dispositions répressives de façon à réprimer la violation des normes prescrites, laquelle constitue alors une infraction. En ce sens, les textes de police fixent, le plus souvent, des sanctions administratives de nature surtout préventive et des sanctions judiciaires de nature surtout répressive.

Mais il convient de garder à l'esprit que l'énoncé d'une valeur limite peut apparaître comme un pis-aller plutôt que comme une solution idéale. En effet, elle n'intègre pas, dans sa logique, une incitation à réduire les niveaux d'exposition en-deçà du niveau maximal requis par la législation, même si cette réduction apparaît techniquement et économiquement envisageable [22]. On peut, en effet, penser dans un premier temps qu'il s'agit là d'une solution satisfaisante et qu'on atteint ainsi le but recherché.

Par rapport à la notion de seuil, le principe d'optimisation oblige les exploitants à faire un effort de réduction des doses tout en sauvegardant la viabilité économique des activités. De ce point de vue, l'énoncé du principe d'optimisation constitue bien une innovation importante par rapport à l'état général des pratiques en matière de normes de sécurité et de protection. II est, en effet, probable que ce soit la première fois dans ces domaines que l'on consente à aller au-delà d'une conception de type juridique des normes matérialisées dans une donnée quantitative exprimant une certaine limite à ne pas dépasser. 


\section{La nature du principe d'optimisation}

\subsection{Une norme-objectif à caractère général}

Le principe d'optimisation se différencie des règles de droit ordinaire qui ressortent du droit administratif classique car il constitue une règle à caractère prospectif fixant un objectif de qualité à atteindre [3]. Cette disposition n'est pas directement applicable : elle indique ce qu'il est souhaitable de faire mais ne dit pas, concrètement, comment il faut le faire. Elle ne fait que définir les obligations des exploitants par l'indication du but ou du résultat qu'ils doivent s'efforcer d'atteindre et c'est en fonction de ce résultat, guidé par lui, que l'exploitant devra choisir les moyens d'y parvenir. En ce sens, le principe d'optimisation s'apparente à une norme objectif. II opère différemment de l'acte réglementaire dont le processus est d'édicter la règle qui constitue le moyen de parvenir au but que l'on s'est fixé.

Dans notre droit, le principe d'optimisation se départit des obligations de prestation généralement mises à la charge de l'exploitant, de la pléthore des règles ou d'instructions toutes plus précises, directives ou techniques les unes que les autres que le pouvoir réglementaire élabore. Cette notion ne se prête pas à un régime d'obligations juridiques strictes et à un langage réglementaire formel, comme des lois ou des règlements qui exigent des règles rigides, définies avec précision. C'est pourquoi une réglementation ne peut faire intervenir l'optimisation que comme une exigence générale, de la souplesse étant au contraire requise dans son application au plan réglementaire, complétée par le recours à des documents d'orientation.

La communication 85-C347-03 du 31 décembre 1985 (1) de la Commission des communautés européennes sur la mise en cuvre des directives du Conseil 80-836-EURATOM, du 15 juillet 1980 (2) et 84-467EURATOM, du 3 septembre 1984 (3), le suggère d'ailleurs de façon claire quand elle énonce que "les principes fondamentaux de justification et d'optimisation des expositions, formulés dans la Publication 26 de la CIPR et repris au titre III, article 6, de la directive de 1980, n'ont à l'évidence qu'une valeur générale, ce dont il y a lieu de tenir compte lors de leur transposition dans les dispositions législatives, réglementaires et administratives nationales". Elle ajoute que "le troisième principe (limitation des doses) peut, quant à lui, être transposé sans restriction dans une législation nationale à caractère contraignant". Ce type d'approche est celui qui a été retenu dans la réglementation française (voir ci-contre).

(1) Communication $85 / \mathrm{C} 347 / 03$, JOCE $n^{\circ}$ C347 du 3.12.85, p. 9.

(2) Directive du Conseil 80/836/EURATOM du 15 juillet 1980 portant modification des directives fixant les normes de base relatives à la protection sanitaire de la population et des travailleurs contre les dangers résultant des rayonnements ionisants, JOCE $\mathrm{n}^{\circ} \mathrm{L} .246 \mathrm{du}$ 17.09.80, 9-12.

(3) Directive du Conseil 84/467/EURATOM du 3 septembre 1984 modifiant la directive 80/836/EURATOM en ce qui concerne les normes de base relatives à la protection sanitaire de la population et des travailleurs contre les dangers résultant des rayonnements ionisants, JOCE n L.265 du 5.10.84, 4-156. 


\section{Transposition du principe d'optimisation en droit français}

La transposition en droit français des directives du Conseil des Communautés européennes ( $n^{\circ} 80 / 836$ du 15 juillet 1980 et $n^{\circ} 84 / 467$ du 4 septembre 1984 explicitées par la communication $n^{\circ} 85 / \mathrm{C} 347 / 03$ du 31 décembre 1985), qui prennent elles-mêmes en compte les recommandations de la Commission internationale de protection radiologique, a été menée à son terme essentiellement par l'adaptation de trois décrets : le décret $n^{\circ}$ 66-450 du 20 juin 1966 relatif aux principes généraux de protection contre les rayonnements ionisants (4) modifié par le décret $n^{\circ} 88-521$ du 18 avril 1988 (5) ; le décret $n^{\circ} 67-228$ du 15 mars 1967 relatif à la protection des travailleurs contre les rayonnements ionisants hors des installations nucléaires de base (6) abrogé et remplacé par le décret $n^{\circ} 86-1103$ du 2 octobre 1986 (7) modifié par le décret $n^{\circ} 88-662$ du 6 mai 1988 (8) ; le décret $n^{\circ} 75-306$ du 28 avril 1975 relatif à la protection des travailleurs contre les rayonnements ionisants dans les installations nucléaires de base (9), modifié par le décret $n^{\circ} 88-662$ du 6 mai 1988 (8).

Le principe d'optimisation fait l'objet d'un simple rappel de principe à l'article 6 du Titre II du décret $n^{\circ} 66-450$ du 20 juin 1966 relatif aux principes généraux de protection contre les rayonnements ionisants qui dispose que "l'exposition des personnes et le nombre des personnes exposées aux rayonnements ionisants doivent, dans la limite des maximums prévus par la réglementation, être aussi réduits que possible". C'est plutôt à une obligation de comportement que sont soumis les exploitants par la réglementation française qui, dans le domaine de la protection des travailleurs contre les dangers des rayonnements ionisants, définit dans les mêmes termes aux articles 4 du décret $n^{\circ} 86-1103$ du 2 octobre 1986 et 8 $\S 2$ du décret $n^{\circ} 75-306$ du 28 avril 1975 modifié comme suit : "Les matériels, les procédés et l'organisation du travail doivent être conçus de telle sorte que les expositions professionnelles individuelles et collectives soient maintenues aussi bas qu'il est raisonnablement possible en-dessous des limites prescrites par le présent décret. A cette fin, les postes de travail exposés font l'objet d'une analyse dont la périodicité est fonction du niveau d'exposition".

La préoccupation de limiter les doses des travailleurs exposés oblige à un effort particulier d'analyse des expositions des postes de travail tant à la conception des matériels et installations qu'en cours d'exploitation.

Un certain consensus existe pour considérer que ce principe doit être envisagé dans le contexte de l'ensemble des exigences imposées à l'exploitant par l'autorité chargée de la réglementation et, dans une certaine mesure, par la nécessité dans laquelle il se trouve d'exploiter son installation d'une manière efficace par rapport aux coûts.

(4) JO du 30 juin 1966.

(5) JO du 6 mai 1988.

(6) JO du 22 mars 1967

(7) JO du 12 octobre 1986, 12995-12335.

(8) JO du 8 mai $1988,6718-6726$ et rectificatif du 30 juillet, p. 9796.

(9) JO du 30 avril 1975. 
Un certain nombre de critiques ont été faites à l'encontre du caractère trop général du principe d'optimisation tel qu'il est transposé dans la réglementation [8]. On lui reproche de créer une situation d'incertitude dans la réglementation pour ceux qui doivent l'appliquer et se conformer à ce principe, qu'il s'agisse tant des exploitants d'installations nucléaires et des utilisateurs des substances radioactives et rayonnements ionisants que des travailleurs exposés. Si l'on introduit ce principe dans la législation, on estime donc nécessaire de définir des données suffisamment claires car, faute de normes ou moyens concrets, l'application pratique est, en fait, dans le vague et c'est finalement le juge qui décide et qui s'érige alors en législateur.

Certains pays comme l'Allemagne, plutôt que d'adopter une approche du type de celle préconisée par le principe d'optimisation, ont choisi de fixer des objectifs en termes de limites, à des niveaux suffisamment bas pour qu'il ne soit pas nécessaire de poursuivre les efforts pour réduire encore les expositions lorsque ces objectifs sont atteints. Si ces objectifs sont suffisamment ambitieux, le principe d'optimisation n'a plus d'intérêt car l'on se trouve presque inévitablement au-dessous du niveau optimal de protection [18].

Cette démarche correspond à estimer que, en application de l'article 161 du Traité Euratom, l'on est en présence, avec le principe d'optimisation, d'une obligation de résultat et non d'une obligation de moyens et qu'on ne peut échapper à prescrire des limites exactes et déterminées, aussi difficile que cela puisse paraître. Le concept de "praticabilité" qui sous-tend le principe d'optimisation est positif quand il encourage innovation et initiative mais non pas lorsqu'il se confond avec une limite à respecter.

\subsection{Obligation de résultat ou obligation de comportement ?}

Les exploitants peuvent-ils, pour autant, garantir que les objectifs fixés seront atteints ? II semblerait qu'ils ne puissent que s'engager à faire tout leur possible pour qu'ils le soient. On le pressent dans l'expression : "Les expositions devront être maintenues au niveau le plus bas que l'on pourra raisonnablement atteindre compte tenu des facteurs économiques et sociaux". Les exploitants s'engagent ici à "faire tous leurs efforts pour", à "agir au mieux" pour des raisons qui tiennent en partie au caractère aléatoire des phénomènes techniques. Sans doute, le principe d'optimisation définit les obligations des exploitants par l'indication du résultat qu'ils doivent s'efforcer d'atteindre, mais il semblerait qu'elles donnent naissance, non pas à des obligations de résultat auxquelles donnent lieu les limites de dose, mais seulement à des obligations de comportement qui obligent le partenaire de l'administration à faire diligence pour atteindre ces résultats $[12,16]$.

II semble que si l'on fixe comme résultat l'obtention d'une bonne qualité et que l'on soit dans l'impossibilité de déterminer de façon précise le 
résultat attendu, l'obligation de résultat soit exclue. Ce qui est le cas du principe d'optimisation pour lequel on obtient des valeurs variables et non absolues puisque l'on tient compte des facteurs économiques et sociaux. Le critère de l'aléa implique que l'exploitant nucléaire n'étant tenu qu'à faire tout son possible pour maintenir les doses en-deçà des doses prescrites, il n'est contraint qu'à une attitude de prudence et de diligence.

Le principe d'optimisation peut être assimilé aux "règles de l'art" qui apparaissent comme des règles de comportement, qu'il s'agisse de comportement d'ordre intellectuel ou d'ordre matériel. Elles sont "constituées tantôt par des prescriptions tout à fait détaillées dictant tel mode d'agir ou telle abstention, tantôt par un appel à la recherche de modalités inspirées de la prudence et de la diligence et propres à favoriser l'évitement de certains inconvénients" [21].

Le principe d'optimisation correspond davantage au cadre de cette seconde solution qui s'apparente à un savoir faire de qualité et doit être compris comme un moyen de contraindre les exploitants à une vigilance optimale. Le comportement est ici l'objet de l'obligation. Cette obligation constitue l'effort constant de l'exploitant tendu vers une fin simplement souhaitée. Comme le but fait partie intégrante de l'obligation de moyens, l'exploitant devra continuer à œuvrer tant que la fin désirée n'est pas atteinte. Le principe d'optimisation est un comportement tendant vers un but envisagé que l'exploitant ne promet pas d'atteindre. Toute obligation est orientée vers une finalité, ce qui ne veut pas dire que l'exploitant soit tenu de réaliser cette fin. II n'est donc pas responsable s'il ne la réalise pas. En effet, en se proposant d'agir avec diligence, l'exploitant n'encourra la charge de la responsabilité que si son attitude est fautive, la faute pouvant être une erreur de conduite quand il y a contradiction manifeste entre les actes et le comportement promis ou une négligence. Le principe d'optimisation exclut donc le régime de la responsabilité objective détachée de la faute.

Ainsi, dans le cas de l'optimisation, toute prestation à la fois obligatoire et déterminée apparaît difficilement concevable, et il convient d'y substituer des normes de comportement qui permettent aux exploitants d'agir au mieux des intérêts de la collectivité et de leurs intérêts dans le milieu concurrentiel où ils se trouvent placés en évitant les contrôles lourds et incessants des pouvoirs publics.

Il faut noter que le droit connaît depuis longtemps l'obligation de comportement avec la gestion en bon père de famille (bonus pater familias) qui correspond à l'obligation imposée à un personnage standard : l'homme raisonnable. II semble bien que l'obligation de l'exploitant, dans le cas de l'optimisation, lui impose la conduite du bon père de famille. L'attitude de celui-ci peut être comparée à l'homme de l'art (ex : médecin) ne pouvant promettre qu'un comportement diligent. 


\section{De l'incitation économique à la responsabilité professionnelle}

Afin d'assurer l'application du principe d'optimisation, plusieurs types d'instruments peuvent être envisagés. Les uns, tels que la taxation [17, $20,23,25]$, qui constituent des moyens classiques se rattachant à une politique de contrainte, paraissent mal adaptés à la situation, les autres s'orientent vers des solutions plus originales et plus "dynamiques", supposant de la part de l'administration et de l'exploitant une réalisation en commun par la politique contractuelle, éventuellement assortie d'avantages financiers, mais surtout par la pratique fondée sur la motivation et la responsabilité professionnelle.

\subsection{La politique contractuelle}

L'efficacité de la mise en œuvre du principe d'optimisation ne repose pas sur le commandement de la puissance publique, mais sur le consentement et l'adhésion des exploitants : l'acte d'autorité semble ici exclu. Le facteur de qualité qu'introduit le principe d'optimisation a un caractère pédagogique et mieux vaut donc convaincre et persuader par une politique contractuelle.

$\mathrm{Au}$ procédés classiques de l'action unilatérale et contraignante, notamment représentés par les réglementations ou par les taxations, doivent être, sans doute, préférées par l'administration des procédures de négociation et d'accords avec les exploitants à qui elle s'adresse et dont elle cherche à obtenir des comportements dans le sens des objectifs optimisés qu'elle souhaite. II s'agit alors d'une "administration concertée" et "contractuelle" [16].

Pour orienter l'exploitant vers certaines directions et certains comportements, les pouvoirs publics pourraient passer avec lui des conventions dites "d'incitation" reposant sur un accord de volonté. On a qualifié ces procédures de "quasi-contrats" [1] et le terme de quasi-contrat doit être pris au sens où on l'entend en droit public pour désigner certaines formes de coopération entre l'administration et les agents économiques. Ceux-ci, qui diffèrent des quasi-contrats de l'article 1371 du Code civil résultant de l'intervention d'une seule volonté, reposent ici sur un accord de volonté provenant d'une véritable négociation.

Cette procédure semble d'autant plus justifiée que, conformément à la doctrine élaborée par la CIPR, on reconnaît que la gestion du risque radiologique ne saurait s'effectuer en référence aux seuls critères scientifiques mais doit s'ouvrir explicitement aux dimensions économiques et sociales inhérentes aux choix collectifs d'une certaine importance. Dans le cas du principe d'optimisation, l'Etat attend de l'exploitant qu'il s'engage à réaliser un programme conforme à l'objectif fixé. Ces contrats s'appliquant à des cas particuliers permettent une négociation à caractère pédagogique et psychologique. 
En théorie, l'Etat peut, de plus, offrir éventuellement son concours, sous forme d'avantages financiers visant soit l'octroi de crédits, de primes, de subventions, soit de dégrèvements fiscaux aux exploitants qui acceptent de participer aux objectifs fixés. Ces méthodes incitatives, au lieu de "punir" l'exploitant qui ne se conforme pas à l'objectif fixé, récompensent l'action conforme par l'octroi d'avantages financiers incitatifs. Mais, en pratique, l'expérience dans le domaine de la pollution classique, par exemple, a montré que ces divers avantages financiers s'avèrent souvent peu efficaces. Ils n'incitent pas vraiment à la réduction de la pollution par les pollueurs : à la limite, ils peuvent constituer des incitations à la corruption pour des pollueurs qui gonfleraient leurs coûts de lutte contre la pollution afin d'obtenir une aide plus importante. Enfin, sur le plan de l'équité, il faut noter que la charge de la lutte anti-pollution est transférée du pollueur à la collectivité.

\subsection{Motivation et responsabilité collective au service de la "culture" du principe d'optimisation}

L'intégration du principe d'optimisation dans un texte réglementaire qui rend son application obligatoire peut servir de base à une politique d'information et surtout de motivation de la part des pouvoirs publics [19]. Cette politique donne alors naissance à des quasi-contrats comparables aux "gentlemen's agreements" du droit international donnant lieu à de simples obligations morales.

Les exploitants, conscients de la rationalité économique que suppose le principe d'optimisation, sont sans doute motivés de facto sans contrepartie financière de l'administration et, en particulier, sans une participation aux dépenses de protection à engager. Le principe d'optimisation étant un point de repère, une référence pour l'entreprise lui rappelant la nécessité de réaliser la meilleure allocation possible des ressources de protection et donc d'éviter les gaspillages, il s'inscrit dans la logique même du management de l'entreprise. Le principe d'optimisation intervient pour corriger les coûts de protection déraisonnables et "rentabilise" les efforts consentis à une gestion rationnelle de la radioprotection.

Le principe d'optimisation, ainsi réintégré à la gestion de l'entreprise, coïncide avec les objectifs économiques des exploitants qui font preuve d'une volonté réelle d'appliquer ce principe afin d'agir au mieux de leurs intérêts dans le milieu concurrentiel où ils se trouvent placés. D'autant que cette volonté trouve aussi sa source dans le souci qu'ont les exploitants de leur "image" auprès du public ainsi qu'auprès de leur personnel par la politique de réduction des doses. Le principe d'optimisation devient un véritable critère dynamique de responsabilité professionnelle dans les activités nucléaires dont le respect est impératif et s'impose de lui-même. C'est par une prise de conscience et une "responsabilisation" collectives que la mise en œuvre du principe d'optimisation est effective. Celle-ci passe essentiellement par une motivation des acteurs : outre la 
contrainte réglementaire, l'impulsion hiérarchique et la sensibilisation du personnel sont déterminantes [19].

Cette sensibilisation à la responsabilité collective en matière de radioprotection passe par une formulation préalable du principe d'optimisation et, ensuite, par une information permanente sur les objectifs et les résultats. Intégrés au plus haut niveau à la politique de l'entreprise, le principe d'optimisation et son esprit doivent aussi faire partie de l'entraînement du personnel.

\section{Conclusion}

Les paragraphes précédents, s'ils apportent un éclairage renouvelé sur les fondements et la portée du principe d'optimisation, n'épuisent cependant pas tous les problèmes juridiques inhérents à son application. II resterait, en effet, à examiner d'une part la question de la résolution des litiges quant à la mise en œuvre effective du principe et, d'autre part, le difficile et controversé problème de la gestion des niveaux de risques résiduels.

Sur le premier point, la réflexion n'a pas réellement apporté jusqu'ici de réponse satisfaisante. Sur quels critères le juge saisi par une des parties peut-il apprécier si une situation est optimisée ou pas ? Peut-on fonder cette appréciation sur des valeurs de références ? Comment ces dernières peuvent-elles être envisagées d'une portée universelle dès lors que chaque situation apparaît comme un cas particulier ? II existe bien là, pour les juristes, un champ à explorer et l'examen de la jurisprudence dans le domaine reste d'une portée assez limitée.

En ce qui concerne la gestion du risque résiduel, il convient de distinguer deux problèmes bien distincts : d'úne part, celui associé à la délimitation du champ d'application de la réglementation [11] qui renvoit à la notion d'exemption et, d'autre part, celui associé à la compensation des effets sanitaires potentiellement imputables aux expositions résiduelles.

La gestion du risque résiduel, comme celle du règlement des litiges, est loin d'être résolue et il est nécessaire de poursuivre la réflexion dans ces domaines avec le souci de maintenir la cohérence avec les principes généraux qui fondent la démarche de l'optimisation.

Au-delà de ces aspects, il semble que le droit administratif qui se profile dans le cas de l'optimisation doit essentiellement faire confiance aux exploitants des installations nucléaires par l'édiction d'une "norme-objectif" et non plus d'une "norme-règle". Ce droit est celui de l'effort commun, encadré par des actes collectifs avec, pour forfait, la confiance, la concertation, les obligations de comportement responsabilisant chacune des parties envers l'autre dans le respect de la santé de l'homme [13]. 
L'avenir des sciences juridiques réside, sans doute, dans la modification de la conception traditionnelle du droit en vue de forcer l'administration à admettre qu'elle n'est pas toujours celle qui sait. Face à la "surproduction juridique" de l'administration donnant lieu à des règles complexes souvent renouvelées et d'application parfois difficile, le principe d'optimisation ouvre la voie vers une substitution de l'intentionalité à la règle, ce qui, en définitive, correspond sans doute mieux à la gestion du risque envisagée comme la recherche de l'acceptable à un moment. donné dans un contexte donné.

Avec l'obligation de comportement, la gestion du risque trouve une finalité parfaitement appropriée à la prospection en avenir aléatoire. D'une part, la technique juridique de l'obligation de comportement laisse, en effet, une plus grande part à l'initiative et à la capacité d'innovation et, d'autre part, elle permet une possibilité d'adaptation continue. Dès lors, en effet, qu'il est admis, d'une part, que les diverses parties s'attachent à réaliser une œuvre commune à travers un acte collectif et que, d'autre part, pour la réalisation de cet objectif commun, chacun n'a consenti qu'à des obligations de comportement, la révision ne peut léser personne. Cette technique juridique sait donc organiser sa propre transformation pour s'adapter à l'évolution technique et ainsi mieux maîtriser l'avenir.

A l'avant garde de la gestion du risque, le principe d'optimisation s'apparente à la recherche d'un compromis fondé sur les critères suivants :

- prudence,

- efficacité économique,

- équité,

- responsabilité collective.

Ces critères traduisent la nature du principe d'optimisation qui est par essence un facteur de qualité de la radioprotection. II suppose une approche multidimensionnelle et exige que la radioprotection soit appréhendée dans une perspective globale. Aux données et au raisonnement scientifique s'ajoutent donc des dimensions éthiques, sociales, économiques et politiques que la CIPR intègre implicitement dans ses recommandations.

Il s'agit, en fait, de l'aboutissement d'une longue maturation qui a mobilisé un effort de réflexion sans précédent dans le domaine de la gestion des risques technologiques. Lauriston $\mathrm{S}$. Taylor affirmait à ce propos en 1980 : "Dès 1957, je déclarais : 'La radioprotection n'est pas seulement affaire de science, c'est une question de philosophie, de morale et d'information au plus haut niveau !' J'avais ajouté, par la suite, 'd'économie, de politique et d'action publique', mais ce sont tous des éléments d'une approche idéologique" [24]. 


\section{RÉFÉRENCES}

[1] ARRIGHI DE CASANOVA E. - Les quasi-contrats du plan. Droit Social, 1965, (juin), p. 341.

[2] CABALLERO F. - Essai sur la notion juridique de nuisance, Thèse de doctorat en droit. Paris : Librairie générale de droit et de jurisprudence, 1981.

[3] CHAPAL Ph. - Recherche sur la notion et le régime des actes juridiques à caractère prospectif, Actual. Jur. Droit Admin. 1968, (juin), 323-334.

[4] COMMISSION INTERNATIONALE DE PROTECTION RADIOLOGIQUE (CIPR). Recommendations (ICRP Publication 26). Oxford : Pergamon press, 1977.

[5] COMMISSION INTERNATIONALE DE PROTECTION RADIOLOGIQUE (CIPR). Cost-benefit analysis in the optimization of radiation protection, a report by Committee 4 (ICRP Publication 37). Oxford : Pergamon press, 1983.

[6] COMMISSION INTERNATIONALE DE PROTECTION RADIOLOGIQUE (CIPR). Optimization and decision making in radiological protection, a report by Committee 4 (ICRP Publication 55). Oxford : Pergamon press, 1988.

[7] COMMISSION INTERNATIONALE DE PROTECTION RADIOLOGIQUE (CIPR). Recommendations (ICRP Publication 60). Oxford: Pergamon press, 1991.

[8] COMMUNAUTES EUROPEENNES, Commission. Radiation protection optimization "As low as reasonably achievable, 2 European seminar, Luxembourg, 1983, 89 Nov. 1983. (JOLIVET A., SINNAEVE J., Eds.) (EUR-9173). Luxembourg : Communautés européennes, 1984.

[9] COMMUNAUTES EUROPEENNES, Commission, NATIONAL RADIOLOGICAL PROTECTION BOARD (NRPB), CENTRE D'ÉTUDE SUR L'EVALUATION DE LA PROTECTION DANS LE DOMAINE NUCLÉAIRE (CEPN). ALARA from theory into practice, joint report, 10 mars 1990. Luxembourg, CEE : 1990, $182 \mathrm{p}$.

[10] FAGNANI F. - Acceptabilité des risques et enjeux sociaux : le cas des normes radiologiques. Paris : Association pour le développement de l'informatique dans les sciences de l'homme, 1977, $128 \mathrm{p}$.

[11] FIKSEL J. - Toward a de minimis policy in risk regulation. Risk analysis, 1985, 5 (4), 257-259.

[12] FROSSARD J. - La distinction des obligations de moyens et des obligations de résultat, Thèse de doctorat en droit. Paris : Librairie générale de droit et de jurisprudence, 1965, $319 \mathrm{p}$.

[13] HAURIOU A. - Le droit administratif de l'aléatoire (Mélanges Louis Trotabas). Paris : Librairie générale de droit et de jurisprudence, 1970, 197-225.

[14] ILARI O. - Development of ICRP recommendations on optimization : past, present and future. In : Advanced seminar on optimization of radiation protection, Ispra courses, 22-26 September 1986, 27 p.

[15] ILARI O. - The application of optimization of protection to regulation and operational practice. In : Radiation protection optimization, advances in practical implementation, European scientific seminar, Madrid, 12-14 September 1988. (Radiation protection, 44, EUR-12469), Luxembourg : CEE, 1989, 55-73.

[16] JACQUOT H. - Recherches sur le régime et la nature juridique des plans français. Orléans, 1967, $237 \mathrm{p}$.

[17] LAKEY J.R.A., LEWIS J.D., Eds. - ALARA. Principles, practice and consequences, Institution of nuclear engineers symposium on ALARA. Quantitative optimization techniques for radiation protection in the nuclear industry, Londres, 18 Sept. 1986. Bristol : Hilger, 1987, 146 p.

[18] LOCHARD J., WEBB G.A.N. - L'application du principe ALARA : un premier bilan. Radioprotection, 198419 (4), 275-294. 
[19] LOCHARD J., LEFAURE C. - Proposition d'organisation et procédure ALARA pour la préparation, le suivi et le retour d'expérience des chantiers de maintenance : application au RGV. Rapport CEPN $n^{\circ} 166$ bis, 1990, $71 \mathrm{p}$.

[20] ORGANISATION DE COOPERATION ET DE DEVELOPPEMENT ECONOMIQUES (OCDE). - Le principe pollueur-payeur. Définition, analyse, mise en œuvre. Paris: OCDE, 1975, $123 \mathrm{p}$.

[21] PENNEAU A. - Règles de l'art et normes techniques. Paris : Librairie générale de droit et de jurisprudence (Bibliothèque de droit privé, 203), 1989, $295 \mathrm{p}$.

[22] PRIEUR M. - Droit de l'environnement (Précis Dalloz). Paris : Dalloz, 1984, $1101 \mathrm{p}$.

[23] PRUD'HOMME R. - Le ménagement de la nature. Des politiques contre la pollution. Paris : Dunod, 1980, $212 \mathrm{p}$.

[24] TAYLOR L.S. - Influence de certains facteurs 'non scientifiques' sur la pratique et les normes de radioprotection. In : International radiation protection association (IRPA). Radiation protection, a systematic approach to safety, Jerusalem, 9-14 March 1980. Oxford : Pergamon press, 1980.

[25] WHIPPLE C., Ed. - De minimis risk (NATO Challenges of modern society, 10 et Contemporary issues in risk analysis 2), Palo Alto : EPRI, 1987, $208 \mathrm{p.}$

\section{TECHNICIEN SUPERIEUR EN RADIOPROTECTION}

\section{$H / F$}

\section{Cette création de poste est une réelle opportunité de carrière.}

Nous sommes une PME récente, spécialisée dans le traitement de déchets nucléaires de faible activité.

Vous prendrez en charge le poste de RESPONSABLE EN RADIOPROTECTION dans sa globalité.

En interface avec le maître-d'ceuvre, vous contribuerez au choix et à la mise en place dans une usine des matériels radioprotection. Vous établirez puis ferez appliquer les procédures et consignes en radioprotection. Vous prévoierez et assurerez les contrôles pour l'environnement et les personnels.

Agé(e) de plus de 30 ans, vous avez :

- un BTS en radioprotection.

- une expérience professionnelle certaine acquise en milieu industriel travaillant pour les centrales nucléaires,

- de bonnes connaissances en AQ et HSCT,

- une habilitation DATR si possible.

- un bon niveau d'anglais.

Vous êtes avenant(e). Vos qualités personnelles sont la curiosité, la rigueur, l'ouverture d'esprit, la disponibilité, elles teront la difference.

Le lieu de travail sera CERGY PONTOISE, 1 ou 2 ans. Ultérieurement, il sera en province au Sud de la LOIRE.

Merci d'adresser votre dossier de candidature : lettre manuscrite, CV, photo et prétentions, sous réf. 92/04 SFRP, à notre conseil ER RESSOURCES HUMAINES - 118, rue de Tocqueville 75017 Paris.

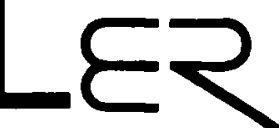

